\title{
Calculation of temperature fields during finishing and strengthening electromechanical treatment of bodies of rotation moving along a helical line by a high-temperature source
}

\author{
Alexander Morozov ${ }^{1, *}$, Lilia Khabieva $^{1}$, Alexey Knyurov ${ }^{1}$, Sergey Petryakov ${ }^{1}$, and Julia \\ Nuretdinova ${ }^{2}$ \\ ${ }^{1}$ Federal State Budgetary Educational Institution of Higher Education Ulyanovsk State Agrarian \\ University, Ulyanovsk, Russian Federation \\ ${ }^{2}$ Federal State Budgetary Educational Institution of Higher Education Ulyanovsk State University, \\ Ulyanovsk, Russian Federation
}

\begin{abstract}
The obtained approximate analytical dependences on the calculation of temperature fields in the processed part during finishing and strengthening electro-mechanical processing allow us to predict the properties of the surface layer and assign processing modes based on the characteristics of operation.
\end{abstract}

\section{Introduction}

Thermal processes occurring during electro-mechanical processing determine the complex of physical and mechanical properties of the surface layer of the processed parts and have a significant impact on the performance of the process. Therefore, the prediction of temperature fields of the surface layer of the processed parts is of considerable theoretical and practical significance. Given the great importance of thermal processes on the nature of Electromechanical processing (EMP), the study of temperature fields in the processed part at EMP is devoted to the work Askinazi B. M. [1], Bagmutov V. P. [2] , etc. Some of the works $[3,4,5]$ are of purely practical significance, in which the influence of technological modes of EMP on the temperature fields of the processed parts is considered. In a number of works [2, 3, 4], the task of theoretical analysis of thermal processes in the processed parts in EMP was set. But, given the complexity of studying thermal phenomena, which are influenced by technological factors of the EMP process, the distribution of heat between contacting bodies, heat exchange with the environment, heat saturation and heat transfer, etc., the theory of thermal processes of EMP is not developed in sufficient depth.

\footnotetext{
* Corresponding author: alvi.mor@mail.ru
} 


\section{Methodology of Researches}

Heat transfer by thermal conductivity is generally described by a parabolic differential equation $[6,7]$.

Taking into account the considerable difficulties in calculating the thermal processes of EMP when a heat source moves on the surface of a cylindrical part along a helical line (Fig. 1) and using the principle of superposition of temperature fields, the inhomogeneous problem is divided into a number of simple ones, and the General solution is found by summing up auxiliary problems.

The heat source makes circular turns on the surface of the cylinder at a distance $\mathrm{S}$ from each other, and the temperature at any point is determined by the total cyclic action of a set of heat sources moving along the axis of symmetry of the part and displaced relative to each other by the value of the longitudinal feed of the tool S. Linear circumferential speed $\mathrm{V}_{0}=\omega \cdot \mathrm{R}$ is significantly greater than the speed of movement of the source $\mathrm{V}$ along the axis of symmetry. The amount of tool feed is several times (3...5) less than the radius (or halfaxis) of the source formed by the contact spot of the processed part and the tool. The following assumptions are made when setting and solving the problem of thermal processes in the processed part in EMP:

1.The Specific heat flow directed from the contact zone to the surface of the part retains a constant value during processing, i.e. the current strength and tool pressure against the part, which determine the heat flow, are unchanged.

2. During the passage of an electric current, the contact resistance causes heat generation to be localized only at the point of contact between the tool and the part.

3 . The temperature in the heating process are the same along the entire cross section of the contact patch.

4. Technological features of the process of forming white (hardened) layers of EMP give reason to believe that significant temperature gradients along the coordinates $\varphi, r$ take place only in the surface layer of the cylinder $\delta$, which is much smaller than the radius of the processed part $\mathrm{R}$.

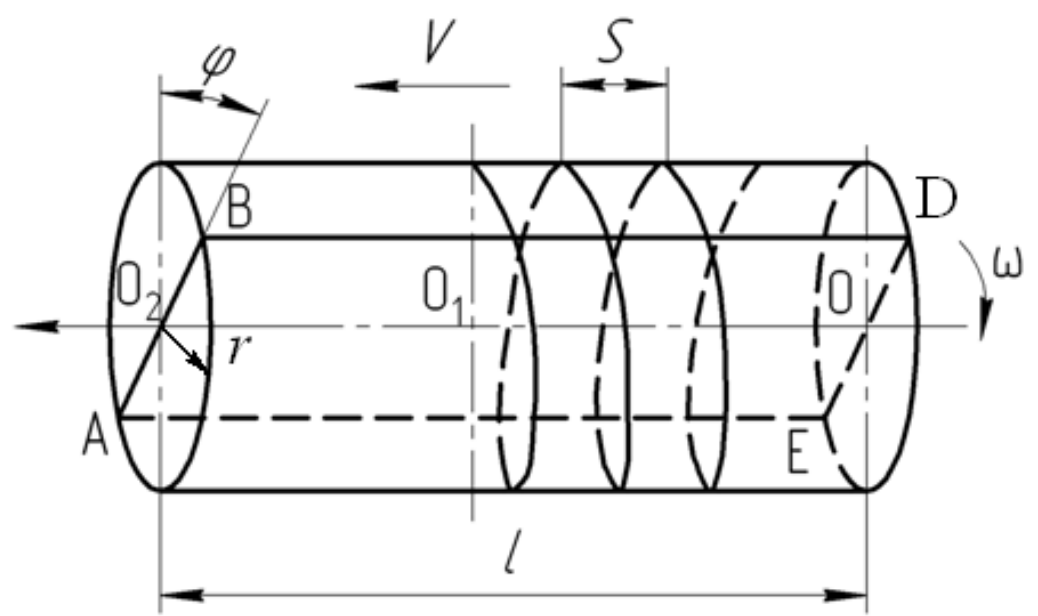

Fig. 1. Diagram of the movement of a heat source in EMP with longitudinal feeding on the surface of a cylindrical part

The stepwise problem of approximate determination of the temperature field of the processed parts in EMP is solved as follows. 
1. heating the cylinder with a heat flow evenly distributed over the surface of the cylinder:

$$
\begin{gathered}
\frac{\partial T_{1}}{\partial t}=a \frac{1}{r} \cdot \frac{\partial}{\partial r}\left(r \frac{\partial T_{1}}{\partial r}\right) \\
0<\mathrm{r}<\mathrm{R}, \mathrm{t}>0, \mathrm{~T}_{1}=(\mathrm{r}, \mathrm{z}, \varphi, 0)=0 \\
-\left.\frac{\partial T_{1}}{\partial r}\right|_{r=R}=\frac{q}{\lambda} ; \mathrm{T}_{1}(0, \mathrm{z}, \varphi, \mathrm{t})<\infty
\end{gathered}
$$

The differential equation is solved (1) by the Fourier variable separation method using the Green formula for the Laplace operator [8]:

$$
T_{1}(r, t)=\frac{q \cdot R}{\lambda}\left[\frac{2 \cdot a \cdot t}{R^{2}}-\frac{1}{4}\left(1-\frac{2 \cdot r^{2}}{R^{2}}\right)-2 \sum_{\kappa=1}^{\infty} \frac{I_{0}\left(v_{\kappa} \frac{r}{R}\right)}{v^{2} \cdot I_{0}\left(v_{\kappa}\right)} \exp \left(-v_{\kappa}^{2} \frac{a \cdot t}{R^{2}}\right)\right],
$$

where $v_{\mathrm{K}}$ - eigenvalues are determined from the characteristic equation $\mathrm{I}_{1}\left(v_{\mathrm{K}}\right)=0$, $\left(v_{\mathrm{K}}\right)=0$.

Where $\mathrm{I}_{1}$ is the first-order Bessel function. When $\frac{a \cdot t}{R^{2}}>0,2$, that is, when approaching the heat saturation, the sum in (3) can be ignored and the temperature can be determined by the formula:

$$
T_{1}(r, t)=\frac{q \cdot R}{\lambda}\left[\frac{2 \cdot a \cdot t}{R^{2}}-\frac{1}{4}\left(1-\frac{2 \cdot r^{2}}{R^{2}}\right)\right]
$$

With a significant change in the coefficients $\lambda$, and from temperature and time, it is impossible to solve equation (1) by the method of separating variables. In this case, it is rational to use one of the approximate methods.

The method of successive approximations of M.E. Schwetz [8] gives the following result (two terms of approximation)

$$
T_{1}=\frac{q}{\lambda}\left[(r-R+\delta)-r \cdot \ln \frac{r}{R-\delta}+\frac{\dot{\delta}}{2 \cdot a}(r-R+\delta)^{2}\right],
$$

where $\delta$ is the depth of significant thermal influence of the heat source on the surface layer of the part (causing phase transformations), is determined from the differential equation:

$$
\delta \dot{\delta}=a\left[1-\ln \left(1-\frac{\delta}{R}\right)\right]
$$

for the initial condition $\delta(0)=0$.

The integral heat balance method allows calculating the temperature field using a simplified, but less accurate, formula: 


$$
T_{1}(r, t)=\frac{q}{2 \cdot \lambda \cdot \delta}(R-\delta-r)^{2}
$$

where the depth of significant thermal influence $\delta$ is determined from the expression:

$$
\delta^{2}\left(1-\frac{1}{4} \cdot \frac{\delta}{R}\right)=6 \cdot a \cdot t
$$

2. The temperature distribution in the cylinder body when rotating with the angular velocity $\omega$ of the flat source $\mathrm{BDOO} 2$, whose temperature is equal to T1 (Fig. 1). the Differential equation has the form:

$$
\begin{gathered}
\frac{\partial T}{\partial t}=a \frac{1}{r^{* 2}} \cdot \frac{\partial^{2} T_{2}}{\partial \varphi^{2}} \\
0<\varphi<2 \cdot \pi, \mathrm{t}>0, \mathrm{~T}_{2}(0)=0 \\
\frac{T_{2}(r, \varphi, t)}{\varphi \quad \omega \cdot t=T_{1}(r, t) ;} \\
\mathrm{T}_{2}(\mathrm{r}, \varphi, \mathrm{t})<\infty
\end{gathered}
$$

Here $r^{*}$ is a fixed radius value. The solution of the differential equation (9) is obtained using the method of thermal potentials of the double layer along the line $\varphi=\omega \cdot t$ :

$$
T_{2}(r, \varphi, t)=\frac{1}{2 \sqrt{a \cdot \pi}} \int_{0}^{t} \frac{r^{*}(\varphi-\omega \cdot t)}{(t-\tau)^{3 / 2}} \exp \left[-\frac{r^{* 2}(\varphi-\omega \cdot t)^{2}}{4 \cdot a(t-\tau)}\right] T_{1}(r, t) d \tau
$$

The solution is obtained on the assumption that the cooling of the cylinder surface occurs very quickly due to significant values of the temperature gradient in the surface layer. This assumption makes it possible to limit the first term when decomposed into a series of potential densities and solve the Volterre equation of the second kind, resulting from condition (10)

$$
\frac{\mathrm{T}_{2}(\mathrm{r}, \varphi, \mathrm{t})}{\varphi}=\omega \cdot t=T_{1}(r, t), \mathrm{t} \geq 0
$$

3. Distribution of temperature in the cylinder body during translational motion with velocity $\mathrm{V}$ along the $\mathrm{Z}$-axis of a circular source, the temperature of which is equal to $\mathrm{T}_{2}$ with the center at the point $\mathrm{O}_{1}$. Taking into account heat transfer, the differential equation of thermal conductivity for this case is written as follows:

$$
\begin{gathered}
\frac{\partial T}{\partial t}=a \cdot \frac{\partial^{2} T}{\partial z^{2}}-s \cdot T \\
\mathrm{z}>\mathrm{z}_{0}+\mathrm{V} \cdot \mathrm{t} ; \mathrm{t}>0 ; \mathrm{T}(\mathrm{z}, 0)=0 ; \mathrm{z} \geq 0 \\
\frac{T(x, r, \varphi, t)}{x \quad}=x_{0}+V \cdot t=T_{2}(r, \varphi, t) ; \mathrm{t}>0 ; \\
\mathrm{T}(\mathrm{z}, \mathrm{r}, \varphi, \mathrm{t})<\infty ; \mathrm{z}>\mathrm{z}_{0}
\end{gathered}
$$

The solution of this differential equation is found in the form of the thermal potential of the double layer along the line $\mathrm{z}=\mathrm{z}_{0}+\mathrm{V} \cdot \mathrm{t}[7,8]$ : 


$$
T(z, r, \varphi, t)=\int_{0}^{t} \frac{\partial G_{0}}{\partial \xi}(z, \xi, t, \tau) \theta(r, \varphi, \tau) d \tau
$$

where $\theta(r, \varphi, t)$ is the density of the potential;

$\xi=z_{0}+V \times t$-coordinate where heat is released;

$\mathrm{G}_{0}$ is the fundamental solution of the thermal conductivity equation or the source Green function for the differential equation (12):

$$
G_{0}=\frac{1}{2 \sqrt{\pi \cdot a^{2}(t-\tau)}} \exp \left[-\frac{(z-\xi)^{2}}{4 \cdot a^{2}(t-\tau)}-\beta(t-\tau)\right]
$$

Substituting the Green function in (14) we find:

$$
T(z, r, \varphi, t)=\frac{1}{4 \sqrt{\pi}} \int_{0}^{t} \frac{z-z_{0}-V \cdot t}{[a(t-\tau)]^{3 / 2}} \exp \left[-\frac{\left(z-z_{0}-V \cdot \tau\right)^{2}}{4 \cdot a^{2}(t-\tau)}-\theta(t-\tau)\right] \theta(r, \varphi, \tau) d \tau
$$

The found function satisfies the equation for $\mathrm{z}>>\mathrm{z}_{0}+\mathrm{V} \times \mathrm{t}$, limited at $\mathrm{z}>>\mathrm{z}_{\text {is } 0}$ and has a zero initial value for any choice of potential density $\theta(t)$.

With $\mathrm{z}=\mathrm{z}_{0}+\mathrm{V} \cdot \mathrm{t}$ the function suffers a break and when

$$
\mathrm{z}=\mathrm{z}_{0}+\mathrm{V}(\mathrm{t}+\varepsilon)
$$

where $\varepsilon$ is an infinitesimal quantity, it has a limit value of $22_{2}(r, \varphi, t)$.

From the condition: $\frac{T(r, \varphi, z, t)}{z} z_{0}+V \cdot t=T_{2}(r, \varphi, t) ; \mathrm{t}>0$ follows:

$$
\frac{\theta(r, \varphi, t)}{2 \cdot a}+\frac{V}{4 \cdot \sqrt{\pi}} \int_{0}^{t} \frac{1}{a^{3 / 2}(t-\tau)^{1 / 2}} \exp \left[-\left(\frac{V^{2}}{4 \cdot a}+\theta\right) \cdot(t-\tau) \theta(r, \varphi, \tau)\right] d \tau=T_{2}(r, \varphi, \tau)
$$

This is an integral Volterra equation of the second kind, from which the potential density $\theta(r, \varphi, t)$ is determined.

In our case, the boundary of the region moves at a low speed and the Volterr equation is simplified. Limiting ourselves to the first term of the potential density expansion in a series, we obtain

$$
\theta(\mathrm{r}, \varphi, \mathrm{t}) \approx 2 \cdot \mathrm{a} \cdot \mathrm{T}_{2}(\mathrm{r}, \varphi, \mathrm{t})
$$

Then the temperature distribution in the cylinder body can be written as:

$$
\begin{aligned}
& T(z, r, \varphi, t)=\frac{q}{\lambda} \cdot \frac{R}{4 \cdot a \cdot \pi} \int_{0}^{t} \frac{z-z_{0}-V \cdot t}{(t-\tau)^{3 / 2}} \exp \left[-\frac{\left(z-z_{0}-V \cdot \tau\right)^{2}}{4 \cdot a^{2}(t-\tau)}-\beta(t-\tau)\right] d \tau \times \\
& \times \int_{0}^{t} \frac{r^{*}(\varphi-\omega \cdot t)}{(t-\tau)^{3 / 2}} \exp \left[-\frac{r^{* 2}(\varphi-\omega \cdot t)^{2}}{4 \cdot a^{2}(t-\tau)}\right] d \tau \times\left[\frac{2 \cdot a \cdot t}{R^{2}}-\frac{1}{4}\left(1-\frac{2 \cdot r^{2}}{R^{2}}\right)\right]
\end{aligned}
$$

The resulting solution allows for the approximate calculation of integrals for determining the temperature of the limit state, the temperature of the surface of the part in the HOU plane, and the calculation of transients under heat saturation. 


\section{Research Results and Discussion}

Figures 2, 3 and 4 show the results of calculations of the temperature field of the limit state $(t \rightarrow \infty)$ of the part within the contact spot with the tool in the coordinate system associated with the heat source. The calculation was made for the case of processing a part made of $40 \mathrm{X}$ steel and copper current-carrying wires.

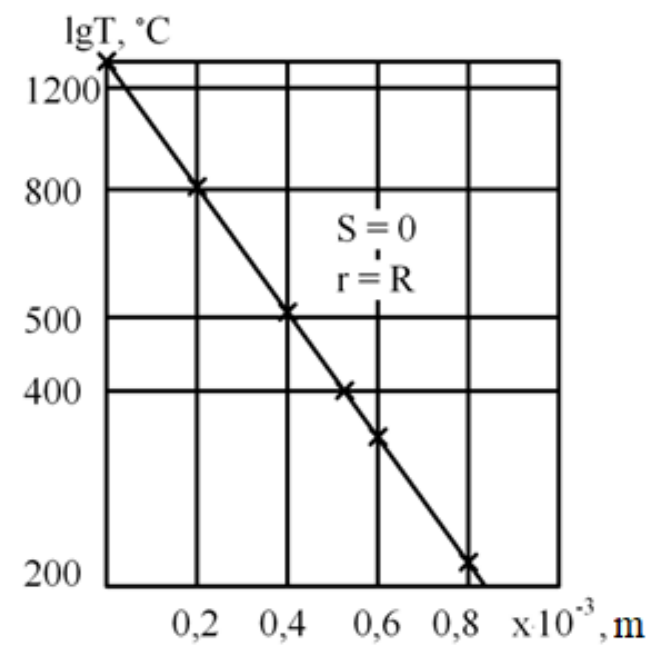

Fig. 2. Temperature field of the limit state (surface heating) of a $40 \times 40$ steel part made of a hard alloy T15K6

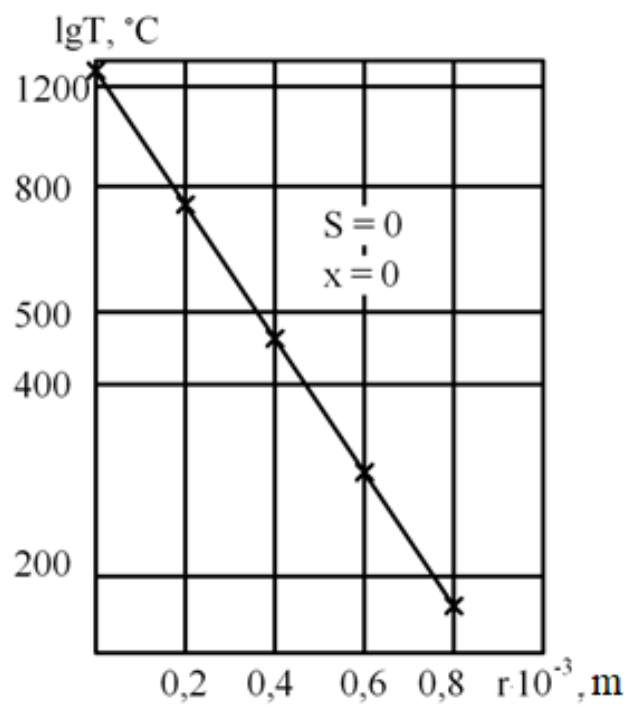

Fig. 3. Temperature field of the limit state of heating of a part made of steel $40 \mathrm{X}$ with a hard alloy tool T15K6 along the axis of action of the maximum temperatures of a normallycircular source 


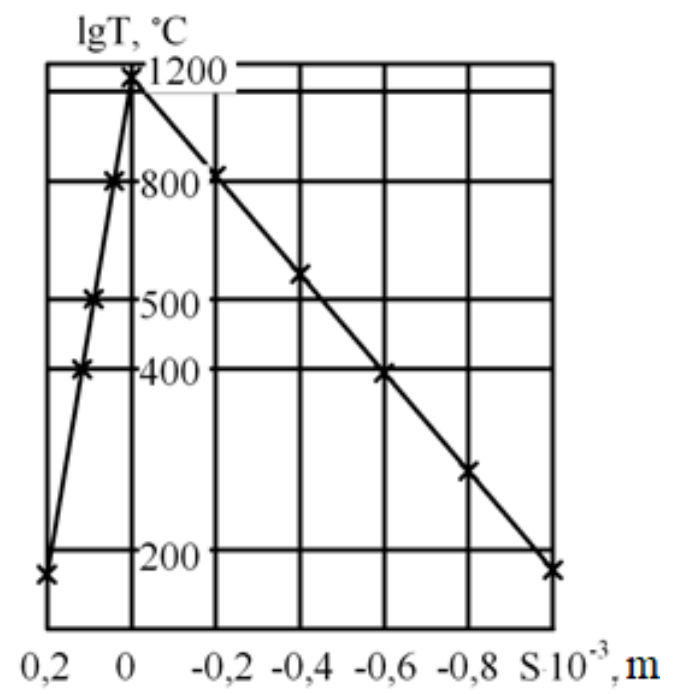

Fig. 4. Temperature field of the limiting state of heating of a part in the HOU plane relative to the center of action of the source: $(+)$ ahead of the source; $(-)$ behind the source

Current strength $\mathrm{I}=400 \mathrm{~A}$; the force of pressing the tool to the workpiece $\mathrm{R}=400 \mathrm{~N}$; the diameter of the workpiece $\emptyset=60 \cdot 10 \mathrm{~m}-3$; the tool feed $\mathrm{S}=0,2 \mathrm{~mm} / \mathrm{rev}$; rotation frequency $\omega=1 \mathrm{~s}-1$; the speed smoothing $\mathrm{V}=0,88 \mathrm{~m} / \mathrm{s}$; coefficient of thermal conductivity of the part material $\lambda=41,89 \mathrm{~V} /\left(\mathrm{m}^{\circ}{ }^{\circ} \mathrm{C}\right)$; the thermal diffusivity a $=$ of $1.07 \cdot 10-5$; the heat transfer coefficient $\alpha=$ of $33.44 \mathrm{~V} /\left(\mathrm{m} 2 \cdot{ }^{\circ} \mathrm{C}\right)$ specific heat capacity $\mathrm{c}=501,6 \mathrm{~J} /\left(\mathrm{kg} \cdot{ }^{\circ} \mathrm{C}\right)$; density of the material, $\gamma=7,8 \cdot 10^{-3} \mathrm{~kg} / \mathrm{m}^{3}$.

A number of ready-made recommendations for integrating and calculating the obtained results are set out in the book by N.N. Rykalin [9]. Thus, for example, the calculation of the temperature of the limit state of the contact point in the HOU plane by the formula for heating a semi-infinite body with a mobile normally circular constant power sourceisdescribed by the equation:

$$
T(r, \varphi, 0, \infty)=\frac{q}{4 \cdot \lambda \sqrt{\pi \cdot a \cdot t_{0}}} \exp (-2 \sqrt{n \cdot p} \cos \varphi) \cdot \frac{1}{\pi} A(0, n, p),
$$

where

$$
A(0, n, p)=2 \int_{0}^{\infty} \frac{d \omega}{1+\omega} \exp \left[-\frac{n}{1+\omega^{2}}-p\left(1+\omega^{2}\right)\right]
$$

alculated using the Simpson formula and for some values $P=\frac{V^{2} \cdot t_{0}}{4 \cdot a}$; $n=\frac{r^{2}}{4 \cdot a \cdot t_{0}}$ presented as graphs.

So, taking into account the distribution of heat from the contact zone to the tool and the workpiece on the technological parameters EMP of steel $40 \mathrm{X}$ by tool from hard alloy T15K6 is defined by heat flux q, the coefficient of concentration of source To and the values And $(0, n, R)$ of $n$ and $R$ at [9]. The limit state temperature is obtained equal to 1232 ${ }^{\circ} \mathrm{C}$, which is in good agreement with the calculations of the center of the contact spot in the limit state for the tool (assuming that the temperatures of the part and the tool in contact are equal) and the calculations of the temperature of the limit state of the part. From figures 2 
and 3, it can be seen that the temperature decreases more slowly in the HOU plane than in the depth of the part, which is explained by the heat sink conditions and the concentration coefficient of the source.

\section{Conclusion}

The given theoretical and experimental results based on the study of temperature fields make it possible to create a mathematical model for the formation of the required operating conditions for the physical and mechanical properties of the surface (hardness, residual stresses, wear resistance, etc.).

\section{References}

1. B.M. Askinasi, Strength improvement and rebuilding of machine parts by using electromechanical hardening (Moscow, Mashinostroenie, 1989)

2. V.P. Bagmutov, S.N. Parshev, N.G. Dudkina, I.N. Zakharov, Electromechanical processing: technological and physical foundations, properties, implementation, (Science, 2003)

3. A.V. Morozov, G.D. Fedotov, K.R. Kundrotas, The influence of volumetric electromechanical mandreling on the lead yield from the matrix material on the bronze bearing bushing surface (Conference Series: Materials Science and Engineering Collection of materials of the International Conference on Modern Trends in Manufacturing Technologies and Equipment: Mechanical Engineering and Materials Science, 2020)

4. A.V. Morozov, G.D. Fedotov, L.V. Fedorova, D.R. Musharapov, L.L. Khabieva, The providing durability of the movable square-sided spline joints by electromechanical treatment of the working surfaces (Matec web of conferences, The proceedings international conference on modern trends in manufacturing technologies and equipment: Mechanical engineering and materials science, 2019).

5. A.E. Abramov, A.V. Morozov, A.O. Koshkina, S.N. Petryakov, Ju.V. Nuretdinova, The cleaning process model of diesel fuel in an electric field (Matec web of conferences, The proceedings international conference on modern trends in manufacturing technologies and equipment: Mechanical engineering and materials science, 2019).

6. A.V. Lykov, Heat and mass transfer. Directory (Moscow, Energiya, 1978)

7. A.N. Tikhonov, A.A. Samarskiy, Equations of mathematical physics (Moscow, "Science", 1977)

8. E.M. Kartashev, Analytical methods in the thermal conductivity of solids (Moscow, Higher school, 1979)

9. N.N. Rykalin, Calculations of thermal processes during welding (Moscow, Mashgiz, 1951) 